\title{
Expression and functional analyses of a Kinesin gene GhKIS13A1 from cotton (Gossypium hirsutum) fiber
}

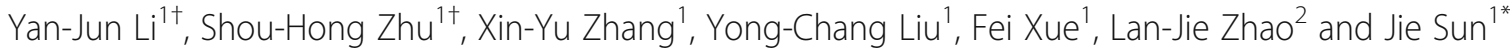

\begin{abstract}
Background: Cotton fiber, a natural fiber widely used in the textile industry, is differentiated from single cell of ovule epidermis. A large number of genes are believed to be involved in fiber formation, but so far only a few fiber genes have been isolated and functionally characterized in this developmental process. The Kinesin 13 subfamily was found to play key roles during cell division and cell elongation, and was considered to be involved in the regulation of cotton fiber development.

Results: The full length of coding sequence of GhKIS13A1 was cloned using CDNA from cotton fiber for functional characterization. Expression pattern analysis showed that GhKIS13A1 maintained a lower expression level during cotton fiber development. Biochemical assay showed that GhKIS13A1 has microtubule binding activity and basal ATPase activity that can be activated significantly by the presence of microtubules. Overexpression of GhKIS13A1 in Arabidopsis reduced leaf trichomes and the percentage of three-branch trichomes, and increased two-branch and shriveled trichomes compared to wild-type. Additionally, the expression of GhKIS13A1 in the Arabidopsis Kinesin-13a-1 mutant rescued the defective trichome branching pattern of the mutant, making its overall trichome branching pattern back to normal.
\end{abstract}

Conclusions: Our results suggested that GhKIS13A1 is functionally compatible with AtKinesin-13A regarding their role in regulating the number and branching pattern of leaf trichomes. Given the developmental similarities between cotton fibers and Arabidopsis trichomes, it is speculated that GhKIS13A1 may also be involved in the regulation of cotton fiber development.

Keywords: Kinesin13 subfamily, Cotton fiber, Trichome, GhKIS13A1

\section{Background}

Cotton is one of the most important commercial crops in the world. Cotton fiber, a natural fiber widely used in the textile industry, is differentiated from single cell of ovule epidermis. The development of cotton fiber consists of four overlapping stages: fiber initiation, cell elongation, secondary wall deposition and maturation [1]. A large number of genes are believed to be involved in fiber formation, but so far only a few fiber genes have been isolated and functionally characterized in this developmental process [2-6]. Therefore, further efforts to

\footnotetext{
* Correspondence: sunjie@shzu.edu.cn

${ }^{\dagger}$ Equal contributors

'The Key Laboratory of Oasis Eco-agriculture, Agriculture College, Shihezi

University, Bei 4 Road, Shihezi 832003, Xinjiang, China

Full list of author information is available at the end of the article
}

clone genes involved in fiber development and study their molecular mechanism governing fiber development are needed. The Kinesin family belongs to a class of motor proteins which can move along microtubule filaments by utilizing the energy released from ATP hydrolysis. Kinesins are present in all studied eukaryotic organisms and are implicated in a diverse range of cellular processes including intracellular transport, signal transduction, cell morphogenesis and mitosis [7-11]. Kinesins can be divided into 14 subfamilies from Kinesin-1 to Kinesin-14 based on the motor domain, a highly conserved domain with the ATP-binding and microtubule-binding activities [12]. The motor domain can be located at the $\mathrm{C}$-terminus, $\mathrm{N}$-terminus or in the middle of Kinesins. With the complete sequencing of many plant genomes, much research has been carried 
out on the Kinesin family in model plants. Sixty-one and forty-one Kinesin genes have been identified in the Arabidopsis and rice genome, respectively [13-15]. To our knowledge, only four Kinesin genes have been reported in cotton (Gossypium hirsutum). GhKCBP, GhKCH1 and GhKCH2 belonging to the Kinesin-14 subfamily were found to decorate microtubules and microfilaments and regulate their alignment and dynamic distribution [16-18]. GhKinesin-13A belonging to the Kinesin-13 subfamily was found to be located in the Golgi apparatus of cotton fiber based on immunofluorescent detection [19]. These studies suggested that Kinesin genes may play key roles in cotton fiber growth and developmental processes.

Much more attention was paid to the Kinesin-13 subfamily recently because of its roles in cell division and cell elongation. Mutation of SRS3 encoding a member of the Kinesin-13 subfamily causes small and round seed phenotype in rice due to a decrease in cell length in the longitudinal direction [20]. T-DNA insertion in the AtKinesin-13A gene results in a sharp decrease of the size and number of Golgi vesicles in root cap peripheral cells [21]. Moreover, it was shown that over $70 \%$ of leaf trichomes in the Kinesin-13a-1 mutants bore four branches, while the trichomes of wild-type (Col-0) predominantly bore three branches [19]. Both cotton fibers and Arabidopsis trichomes are derived from single cells of epidermis, and likely to be regulated by a similar development mechanism. Therefore, genes that influence the development of Arabidopsis trichome may also play key roles in cotton fiber development. In a previous study, we isolated seven putative G. hirsutum homologues of AtKinesin$13 A$ using primers designed based on the genome sequence of $G$. raimondii, and analyzed the sequence characteristics of these Kinesin genes [22]. Of the seven Kinesin-13 genes, GhKIS13A1 was found to be virtually identical to the previously reported GhKinesin-13A, a homologue of the Arabidopsis AtKinesin-13A [19]. In this study, on the basis of expression pattern and biochemical characterization of GhKIS13A1, we showed that GhKIS13A1 not only influences the number and branching patterns of Arabidopsis leaf trichomes but also is able to rescue the mutant phenotype of the Arabidopsis Kinesin-13a-1 mutant, suggesting that GhKIS13A1 may play an important role in cotton fiber development and is a potential candidate for improving cotton fiber quality by genetic engineering.

\section{Methods}

\section{Materials}

Flowers and fibers were collected from the cotton (Gossypium hirsutum) variety "Xinluzao 36" plants grown in a normal agronomic field. Flower buds on the anthesis day were tagged and marked as 0 day post anthesis (DPA). Bolls were collected at 3, 6, 9, 12, 15,
18, 21, 24 and 27DPA. Ovules were excised from the bolls and fibers were scraped from the ovules. The seeds of "Xinluzao 36" were sterilizated by $0.1 \% \mathrm{HgCl}_{2}$ for $10 \mathrm{~min}$, washed with sterile water 3-4 times and then sown on the Murashige and Skoog (MS) ager medium. The root, hypocotyl and leaf were collected from the tissue-cultured seedlings grown for about 2 weeks. All the collected materials were immediately frozen in liquid nitrogen and stored at $-80^{\circ} \mathrm{C}$ until RNA extraction. The ecotype Columbia (Col-0) of Arabidopsis thaliana and the Kinesin-13a-1 mutant were used in transgenic analysis.

\section{Cloning of GhKIS13A1}

Total RNAs were extracted from cotton fibers and then reverse transcribed to generate cDNA. Gene-specific primers KIS-1: 5'-ATG GGT GGC CAG ATG CAG CAA AGC-3' and KIS-2: 5' ${ }^{\prime}$ ACG AGG AAC TCT TTT CCG ACT C-3' were designed based on the GhKIS13A1 sequence (accession number, KP036626) downloaded from the GenBank database. The open reading frame (ORF) of GhKIS13A1 was amplified by PCR using the gene-specific primers and cotton fibers cDNA as a template. The PCR amplification program was: $94^{\circ} \mathrm{C}$ for $3 \mathrm{~min}, 30$ PCR cycles $\left(94^{\circ} \mathrm{C}\right.$ for $1 \mathrm{~min}, 56^{\circ} \mathrm{C}$ for $1 \mathrm{~min}$ and $72^{\circ} \mathrm{C}$ for $2.5 \mathrm{~min}$ ), and $72^{\circ} \mathrm{C}$ for $10 \mathrm{~min}$. The PCR product was purified and cloned into the pGEM-T easy vector (Promega, USA), and then transformed into $E$. coli DH10B cells for sequencing.

\section{Phylogenetic analysis}

Kinesin proteins from Arabidopsis, rice, and corn were obtained from the GenBank database (http://www.ncbi.nlm. nih.gov/genbank/). Thirteen cotton Kinesins were obtained from the genome database of Gossypium hirsutum (www.cottongen.org/) by using GhKIS13A1 as a probe. MEGA 4.0 was used for the construction of phylogenetic tree, using the neighbor-joining method and the bootstrap test carried out with 1000 replicates.

\section{Expression pattern of GhKIS13A1 in different cotton organs}

Total RNAs from cotton roots, hypocotyls, leaves, flowers, ovules and fibers were extracted according to a previous method [23], and reversely transcribed into cDNAs. Then, the cDNAs were used as templates in Real-time Quantitative PCR (qPCR) reactions with genespecific primers. The forward primer KIS-5 is $5^{\prime}-\mathrm{CTG}$ GTC GAA GGG TAG CAG AG-3', and the reverse primer KIS-6 is $5^{\prime}$-GGC TCG AAG AAC CAC CAT AA-3'. The cotton $U B I$ gene was used as an internal reference for normalization of cDNA templates. The forward primer of $U B I$ is $5^{\prime}$-CAG ATC TTC GTA AAA CCC T-3', and the reverse primer is $5^{\prime}$-GAC TCC TTC TGG ATG TTG TA-3'. The qPCR reactions were 
conducted using a SYBR Green I Master mixture (Roche, Switzerland) on a LightCycler 480IIsystem (Roche, Switzerland) under conditions of an initial denaturation at $95{ }^{\circ} \mathrm{C}$ for 2 min followed by 40 cycles of denaturing at $95^{\circ} \mathrm{C}$ for $15 \mathrm{~s}$, annealing at $55^{\circ} \mathrm{C}$ for $20 \mathrm{~s}$, and extending at $72{ }^{\circ} \mathrm{C}$ for $15 \mathrm{~s}$. The qPCR reaction was repeated three times. Quantification was performed using the $2^{-\triangle \Delta \mathrm{Ct}}$ method.

\section{Expression and purification of the recombinant GhKIS13A1 protein}

The GhKIS13A1 ORF was cloned into plasmid pET28a (+) (with a His tag) using EcoR1/HindIII restriction sites to generate the pET28a-GhKIS13A1 construct. After sequencing confirmation, the pET28a-GhKIS13A1 construct was transformed into E. coli BL21 (DE3) cells for fusion protein expression. A single colony of E.coli BL21 cells harboring the recombinant plasmid pET28aGhKIS13A1 was incubated at $37{ }^{\circ} \mathrm{C}$ until the $\mathrm{OD}_{600}$ reached about 0.6 , and protein synthesis was induced by adding $0.8 \mathrm{mM}$ isopropyl thio- $\beta$-D-galactoside (IPTG) for up to $6 \mathrm{~h}$ at $37^{\circ} \mathrm{C}$. Protein purification was performed with His-Binding-Resin following the manufacturer's instructions (Yuekebio, Shanghai, China). The purity of the GhKIS13A1-His fusion protein was loaded on $12 \%$ SDS-PAGE gel for western blot experiment with anti$6 \times$ His antibody (Sigma). The protein concentration was determined by the Bradford method with bovine serum albumin (BSA) as the standard.

\section{Assays for Kinesin activity of GhKIS13A1}

Microtubule binding assay was performed according to a previous method [24]. To prepare microtubules, the tubulin protein (GenMed Scientifics Inc. USA) was polymerized by incubating with $2 \mathrm{mmol} \cdot \mathrm{L}^{-1}$ GTP and $30 \mu \mathrm{mol} \cdot \mathrm{L}^{-1}$ paclitaxel for $30 \mathrm{~min}$ at $37^{\circ} \mathrm{C}$. A volume of $50 \mu \mathrm{l}$ microtubules $\left(2.7 \mathrm{mg} \cdot \mathrm{mL}^{-1}\right)$ was mixed with $50 \mu \mathrm{l}$ purified GhKIS13A1 $\left(0.44 \mathrm{mg} \cdot \mathrm{mL}^{-1}\right)$ in the absence or presence of $10 \mathrm{mmol} \cdot \mathrm{L}^{-1} \mathrm{ATP}$ and $5 \mathrm{mmol} \bullet \mathrm{L}^{-1} 5$-adenylylimidodiphosphate (AMP-PNP; a non-hydrolyzable ATP analogue). After incubation for $15 \mathrm{~min}$ at $37^{\circ} \mathrm{C}$, the reactions were centrifuged at $14,000 \mathrm{~g}$ for $30 \mathrm{~min}$. The supernatant and pellet were electrophoresed by SDSPAGE and stained with Coomassie Brilliant Blue R-250.

To analyze the ATPase activity and microtubuleactivated ATPase activity, $100 \mu \mathrm{l}$ purified GhKIS13A1 protein $\left(0.44 \mathrm{mg} \cdot \mathrm{mL}^{-1}\right)$ was incubated with $100 \mu \mathrm{l}$ microtubulins $\left(2.7 \mathrm{mg} \cdot \mathrm{mL}^{-1}\right)$ for $15 \mathrm{~min}$ at $37^{\circ} \mathrm{C}$. Then $20 \mu \mathrm{l}$ ATP $\left(20 \mathrm{mmol} \cdot \mathrm{L}^{-1}\right)$ was added and incubated for another $15 \mathrm{~min}$. The reaction was terminated by adding $20 \mu \mathrm{l}$ of $100 \%$ trichloroacetic acid followed by placing on ice for $10 \mathrm{~min}$. After $10 \mathrm{~min}$ of centrifugation at $4^{\circ} \mathrm{C}$, the supernatant was collected and used to determinate the inorganic phosphate according to the LeBel's method [25].

\section{Vector construction and Arabidopsis transformation}

The GhKIS13A1 ORF was amplified using KIS-1 and KIS-2 primers and then constructed into plant expression vector pGWB17 (with a $35 \mathrm{~S}$ promoter) by the Gateway technology. To ensure the sequence conformity to the entry vector in the right direction in the process of vector construction, the recognition sequence 'CACC' was added to the 5' end of the forward primer KIS-1. The PCR product was integrated into the entry vector pENTR/D-TOPO following the instructions of pENTR $^{\mathrm{Tm}}$ Directional $\mathrm{TOPO}^{\circ}$ Cloning Kits (Invitrogen, USA). After confirmed by DNA sequencing, the entry vector carrying the GhKIS13A1 ORF was mixed with expression vector pGWB17 for LR recombination reaction following the in${\text { structions of Gateway }{ }^{\circ} \text { LR Clonase }}^{\mathrm{Tm}}$ II Enzyme Mix (Invitrogen, USA). The confirmed plasmid was transformed into Agrobacterium tumefaciens GV3101 by the standard transformation method [26]. Arabidopsis ecotype Col-0 and the Kinesin-13a-1 mutant were used in transformation by the floral dip method [27] to generate transgenic lines overexpressing GhKIS13A1 (GhKIS13A1-OX) and complementing the Kinesin-13a-1 mutation (GhKIS13A1$\mathrm{COM})$, respectively.

PCR and qPCR detection of Arabidopsis transgenic plants Genomic DNA was extracted from Arabidopsis leaves and used in PCR analysis for detection of the presence of the inserted GhKIS13A1 sequence. The gene-specific primers KIS-1 and KIS-2 were used to detect exogenous GhKIS13A1 transformations. PCR-positive plants harboring the GhKIS13A1 gene were further analyzed for its expression level by qPCR. Total RNA was extracted from 9-dayold wild-type, transgenic lines and the Kinesin-13a-1 mutant using TRIZOL Reagent (Tiangen, China), and reversely transcribed into cDNAs. Then, the cDNAs were used as templates in qPCR reactions with gene-specific primers KIS-5 and KIS-6. The Arabidopsis ACTIN2 gene was used as an internal reference for normalization of cDNA templates. The forward primer of ACTIN2 is $5^{\prime}$-GGT AAC ATT GTG CTC AGT GGT GG, and the reverse primer is 5'-AAC GAC CTT AAT CTT CAT GCT GC-3'.

\section{Analysis of leaf trichomes of Arabidopsis transgenic plants} Six GhKIS13A1-OX and GhKIS13A1-COM transgenic lines, each with seven randomly selected plants, were used in leaf trichome analysis. The leaf of the same part on each plant was picked for counting the total number of trichomes and calculating the percentages of trichomes with different number of branches.

\section{Results}

Analysis of phylogenetic relationships

In the genome database of Gossypium hirsutum, thirteen Kinesin 13 proteins were identified by using the protein 
sequence of GhKIS13A1 as a probe. Along with sequences from Arabidopsis, rice and corn, a phylogenetic tree was constructed (Fig. 1). These proteins from other plants are members of either the Kinesin-13 subfamily or the other subfamilies, including Kinesin-1, Kinesin-2, Kinesin-7, Kinesin-12 and Kinesin-14 subfamilies. Phylogenetic analysis showed that all cotton Kinesins were clustered to Kinesin-13 subfamily, and divided into Kinesin-13A and Kinesin-13B, which were close to AtKinesin-13A and AtKinesin-13B, respectively (Fig. 1). GhKIS13A1 was close to GhKinesin-13A and GhA09G0604 in Kinesin-13A subclade. BLAST searches revealed that the GhKIS13A1 showed 99.56\% identity with previously reported GhKinesin-13A [19], and 99.45\% identity with GhA09G0604 identified from cotton database. These suggest that GhKIS13A1 was virtually identical to GhKinesin-13A and GhA09G0604, located on

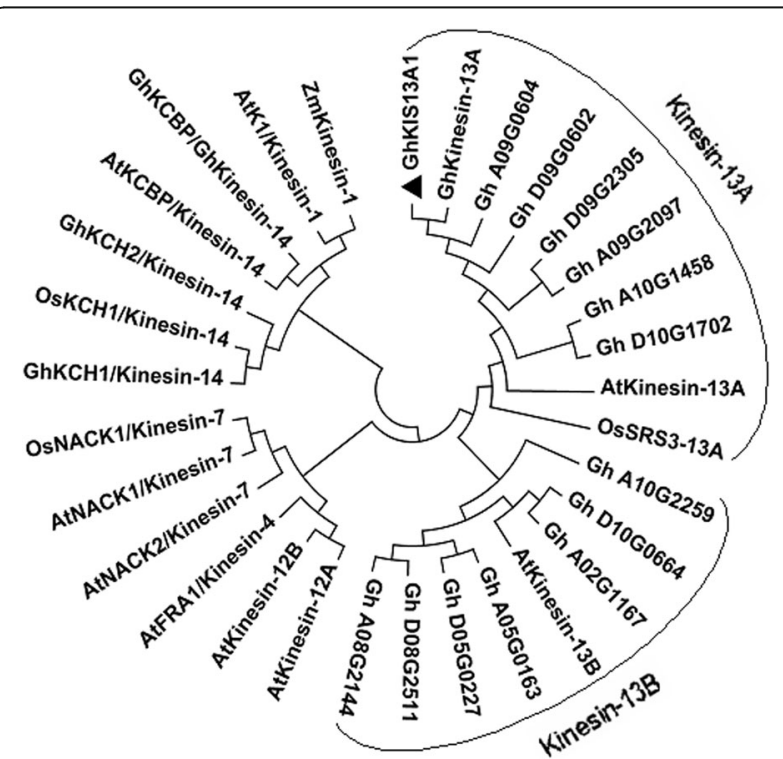

Fig. 1 Phylogenic relationship of GhKIS13A1 and other Kinesin proteins. The phylogenetic tree was constructed by MEGA 4.0 using the neighbor-joining method and the bootstrap test carried out with 1000 bootstrap replicates. The sequence used are GhKIS13A1 (Gossypium hirsutum, KP036626), GhKinesin-13A (Gossypium hirsutum, AAQ18797), AtKCBP/Kinesin-14 (Arabidopsis thaliana, AAC37475), GhKCBP/GhKinesin-14 (Gossypium hirsutum, AAP41107), AtKinesin-13A (Arabidopsis thaliana, AAL07208), AtKinesin-13B (Arabidopsis thaliana, AAK96543), AtKinesin-12A (Arabidopsis thaliana, AAF78893), AtKinesin-12B (Arabidopsis thaliana, AEE76799), AtK1/Kinesin-1 (Arabidopsis thaliana, BAC03248), OsNACK1/ Kinesin-7 (Oryza sativa, BAB86283), GhKCH1/Kinesin-14 (Gossypium hirsutum, AAW03152), GhKCH2/Kinesin-14 (Gossypium hirsutum, ABO28522), OsSRS-13A (Oryza sativa, EEE62380), ZmKinesin-1 (Zea mays, DAA35856), OsKCH1/Kinesin-14 (Oryza sativa, NP_001066967), AtFRA1/Kinesin-4 (Arabidopsis thaliana, AAN86114), and 13 Kinesins from cotton genome database by using GhKIS13A1 as a probe, including Gh_D09G0602, Gh_A09G0604, Gh_A09G2097, Gh_D09G2305, Gh_D10G1702, Gh_A10G1458, Gh_A10G2259, Gh_D10G0664, Gh_A05G0163, Gh_D05G0227, Gh_D08G2511, Gh_A08G2144, Gh_A02G1167 the A09 chromosome. The sequence differences among GhKIS13A1, GhKinesin-13A and GhA09G0604 may be caused by the difference of cotton varieties and the unavoidable small errors during sequencing or sequence assembly.

\section{Expression analysis of GhKIS13A1}

The qPCR was performed to investigate the expression pattern of GhKIS13A1 in different cotton organs. The result showed that GhKIS13A1 was expressed preferentially in flower and leaf, and at a lower level in hypocotyl, root and fiber. In contrast, GhKIS13A1 was expressed at the lowest level in 6-15 DPA fiber cells. Although the expression of GhKIS13A1 was observed at a slightly higher level in 3 DPA ovule and 18-27DPA fiber cells, the level were significantly lower than in the flower and leaf. In addition to 24DPA fiber cell, the expression level of GhKIS13A1 in fiber cells was lower than in the hypocotyl (Fig. 2).

\section{Biochemical characterization of the motor domain in GhKIS13A1}

To carry out the biochemical characterization of GhKIS13A1, the GhKIS13A1 ORF was amplified and ligated into plasmid pET-28a $(+)$ and expressed in E.coli BL21 cells. The GhKIS13A1-His fusion protein prepared using E.coli was tested in vitro for its ATPase activity and microtubulin binding activity. SDS-PAGE showed the IPTG induced production of a $110 \mathrm{kDa}$ protein (Fig. 3a), consistent with the predicted molecular mass of GhKIS13A1-His. The recombinant enzyme GhKIS13A1His was purified by His-Binding-Resin purification column. Western blot experiment showed that GhKIS13A1-His protein could be recognized by his-antibody (Fig. 3b).

To define the ATPase activity and microtubuleactivated ATPase activity, the purified GhKIS13A1-His protein was incubated with ATP in the absence or presence of polymerized microtubulins (MTs). As shown

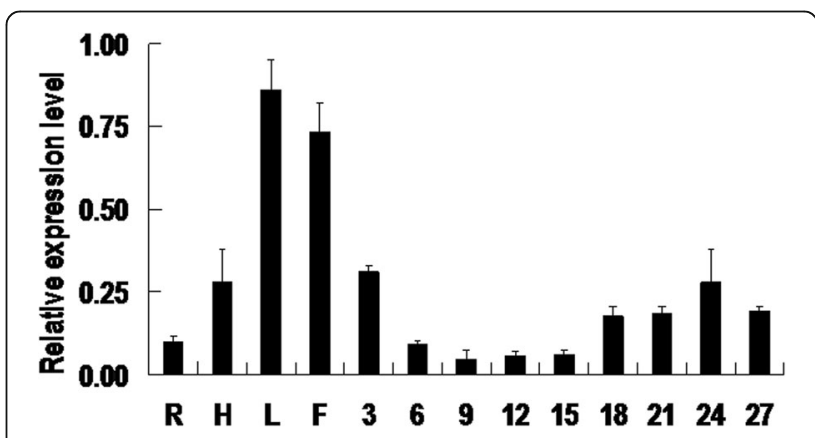

Fig. $\mathbf{2}$ Expression pattern of GhKIS13A1 in different cotton organs. All expression levels are relative to the cotton $U B /$ gene that was used as an internal control. R: root; H: hypocotyl; L: leaf; F: flower; 3: 3DPA ovule; 6-27: the fiber cells at 6, 9, 12, 15, 18, 21, 24 and 27 DPA, respectively 


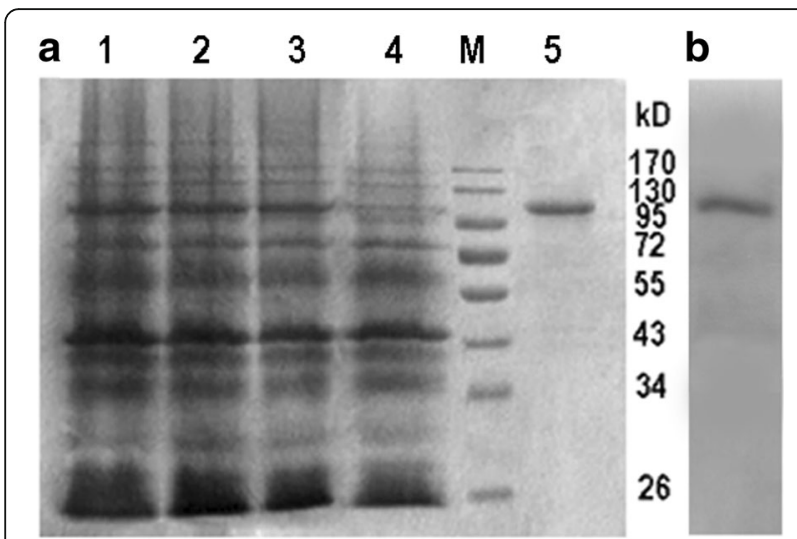

Fig. 3 SDS-PAGE analysis of the induction and purification of GhKIS13A1 expressed in E.coli BL21. a, SDS-PAGE analysis of induction and purification of GhKIS13A1-His fusion protein. M: protein molecular marker; Lane 1: total proteins of cells after $2 \mathrm{~h}$ of IPTG induction; lane 2: total proteins of cells after $4 \mathrm{~h}$ of IPTG induction; lane 3: total proteins of cells after $6 \mathrm{~h}$ of IPTG induction; lane 4: total proteins of cells without IPTG induction; lane 5: purified GhKIS13A1-His fusion protein. b, GhKIS13A1-His fusion protein confirmed by western blot experiment with anti- $6 \times$ His antibody

in Fig. 4, the ATPase activity of GhKIS13A1 was $15.42 \pm$ $1.56 \mathrm{nmol} \cdot \mathrm{Pi} \cdot \mathrm{Min}^{-1} \cdot \mathrm{mg}^{-1}$ in the absence of MTs, and increased by approximately 3 -fold to $56.48 \pm 3.15 \mathrm{nmol} \bullet$ $\mathrm{Pi} \bullet \mathrm{Min}^{-1} \cdot \mathrm{mg}^{-1}$ in the presence of MTs. No ATPase activity was detected in the reaction system containing MTs and lacking GhKIS13A1-His. The result suggested that GhKIS13A1 has a basal ATPase activity that can be activated significantly by the presence of MTs.

To determine the microtubulin binding activity of GhKIS13A1, the purified GhKIS13A1-His protein was incubated with MTs in the absence or presence of either ATP or AMP-PNP, a non-hydrolyzable ATP analogue. In the absence of MTs, the GhKIS13A1-His protein

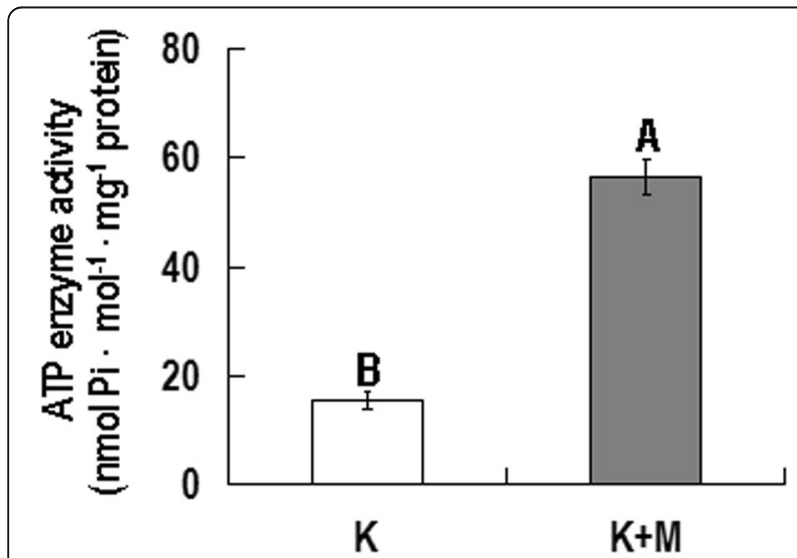

Fig. 4 ATPase activity assays of GhKIS13A1. K: ATPase assays of GhKIS13A1 in the absence of MTs; K + M: ATPase assays of GhKIS13A1 in the presence of MTs. Data were analyzed by Paired-samples $T$ test. Different uppercase letters indicate a significant difference between $\mathrm{K}$ and $\mathrm{K}+\mathrm{M}$ at the $1 \%$ probability level remained in the supernatant after 30 min of centrifugation (Lanes 1-2 of Fig. 5). In the presence of MTs, the GhKIS13A1-His protein was co-precipitated with MTs only in the presence of AMP-PNP but not in the absence of AMP-PNP (comparing Lanes 5-6 and Lanes 7-8 of Fig. 5). These results suggest that AMP-PNP can promote the binding between GhKIS13A1 and MTs, and that GhKIS13A1 has the nucleotide-dependent microtubulin binding ability.

\section{Screening for GhKIS13A1 transgenic Arabidopsis plants}

The GhKIS13A1 overexpression vector was constructed and introduced into the Arabidopsis wild-type (Col-0) and the Kinesin-13a-1 mutant plants to generate GhKIS13A1OX and GhKIS13A1-COM lines, respectively. Successful transgenic plants were selected using PCR based on the presence of the exogenous GhKIS13A1 gene. The T2 generation plants of three GhKIS13A1-OX lines (L1, L2 and L8) and three GhKIS13A1-COM lines (C3, C7 and C10) were selected for leaf trichome investigation. The expression levels of GhKIS13A1 in these independent transgenic lines were quantified using qPCR. As expected, no expression of GhKIS13A1 was detected in the wild-type (Col-0) and the Kinesin-13a-1 mutant, while expression of GhKIS13A1 was detected in the three GhKIS13A1-OX lines (L1, L2 and L3) and three GhKIS13A1-COM lines (C3, C7 and C10) (Fig. 6). Among the three GhKIS13A1OX lines, L2 showed the highest transcript level of GhKIS13A1, followed by the L1 and L8 lines (Fig. 6b). Among the three GhKIS13A1-COM lines, C3 showed the highest transcript level of GhKIS13A1, followed by the C7 and $\mathrm{C} 10$ lines (Fig. 6c). Exogenous expression of GhKIS13A1 in Arabidopsis had no effect on the overall growth and development of the transgenic plants.

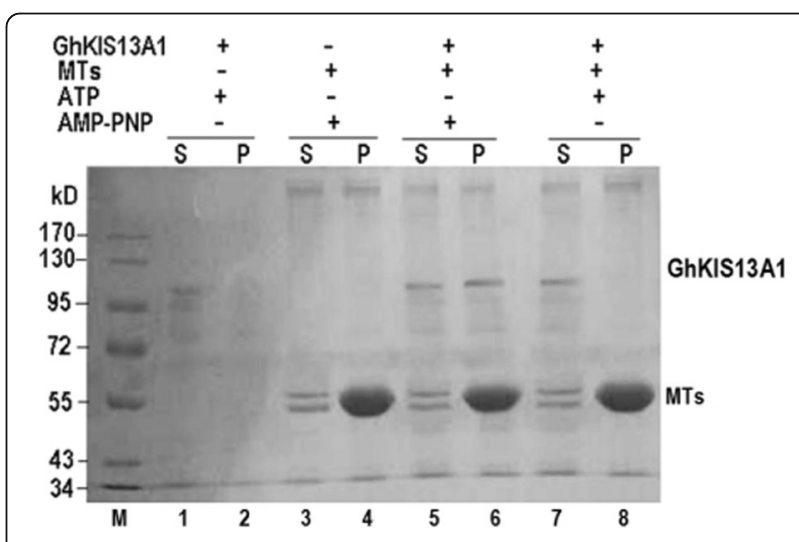

Fig. 5 Microtubule binding assay of GhKIS13A1. M: protein molecular marker; S: supernatant; P: precipitation; lanes 1-2: microtubule binding assays of GhKIS13A1 in the absence of MTs (control test); lanes 3-4: microtubule binding assays of MTs in the absence of GhKIS13A1 (control test); lanes 5-6: microtubule binding assays of GhKIS13A1 in the presence of AMP-PNP; lanes 7-8: microtubule binding assays of GhKIS13A1 in the presence of ATP 


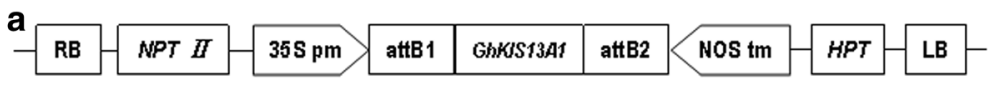

b

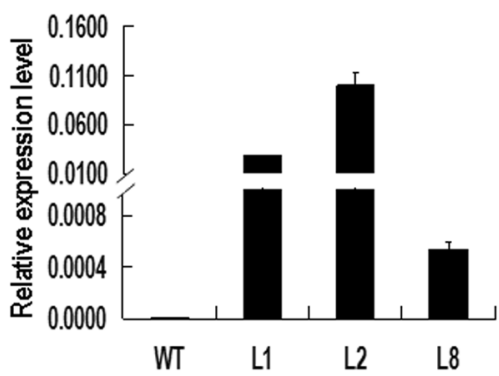

C

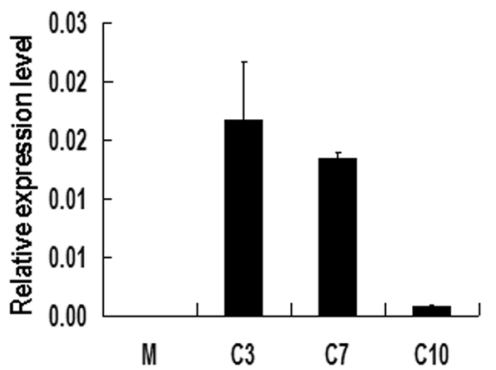

Fig. 6 The PCR and $\mathrm{qPCR}$ detection of transgenic plants. a, Schematic representation of the expression cassettes in the plant expression vector pGWB17-GhKIS13A1 used in Arabidopsis transformation. 35S pm, CaMV 355 promoter; NOS tm, NOS terminor; HPT, hygromycin phosphotralsferase gene; NPT II, neomycin phosphotransferase llgene; attB1 and attB2, recombination site sequences in the Gateway system; GhKIS13A1, cDNA sequence of the GhKIS13A1 gene; RB, right border; LB, left border; (b), The expression level of GhKIS13A1 in wild-type (Col-0) and three GhKIS13A1-OX lines (L1, L2 and L8). WT, wild-type; (c), The expression level of GhKIS13A1 in the Kinesin-13a-1 mutant and three GhKIS13A1-COM lines (C3, C7 and C10). M, Kinesin-13a-1 mutant. All expression levels are relative to the Arabidopsis ACTIN2 gene that was used as an internal control

Overexpression of GhKIS13A1 influences the number and branching of the Arabidopsis leaf trichomes

A previous study showed that AtKinesin-13A plays a role in trichome morphogenesis, and that its Kinesin-13a-1 mutant has more trichomes with four branches than wild-type [19]. Therefore, we compared the number and branching pattern of leaf trichomes between the wildtype and the transgenic lines. The eighth rosette leaves taken from seven plants of each transgenic line were used in the comparison.

The number of trichomes of the three GhKIS13A1-OX lines were significantly less than that of the wild-type (Fig. 7), and was negatively correlated with the expression level of GhKIS13A1 in the corresponding GhKIS13A1-OX line. Line L2, with the highest GhKIS13A1 expression level (Fig. 6), produced 52 trichomes per leaf, a 54.9\% reduction compared with the wild-type (116 trichomes per leaf); line L8, with the lowest expression level of GhKIS13A1, produced 92 trichomes per leaf, which is $20.4 \%$ less than that of the wild-type. Regarding the branching pattern, the trichomes of the wild-type predominantly had three branches (94.7\%), although trichomes with four branches were occasionally seen (4.2\%). In the GhKIS13A1-OX lines, although the majority of trichomes also had three branches, their percentages were significantly lower than that of the wild-type (Table 1). Notably, the transgenic lines produced more two-branch trichomes and shriveled-branch trichomes, suggesting that ectopic expression of GhKIS13A1 in Arabidopsis has a negative effect on branching of trichomes with more than two branches. These data collectively indicated that GhKIS13A1 overexpression can influence the number and branching of the leaf trichomes in Arabidopsis.
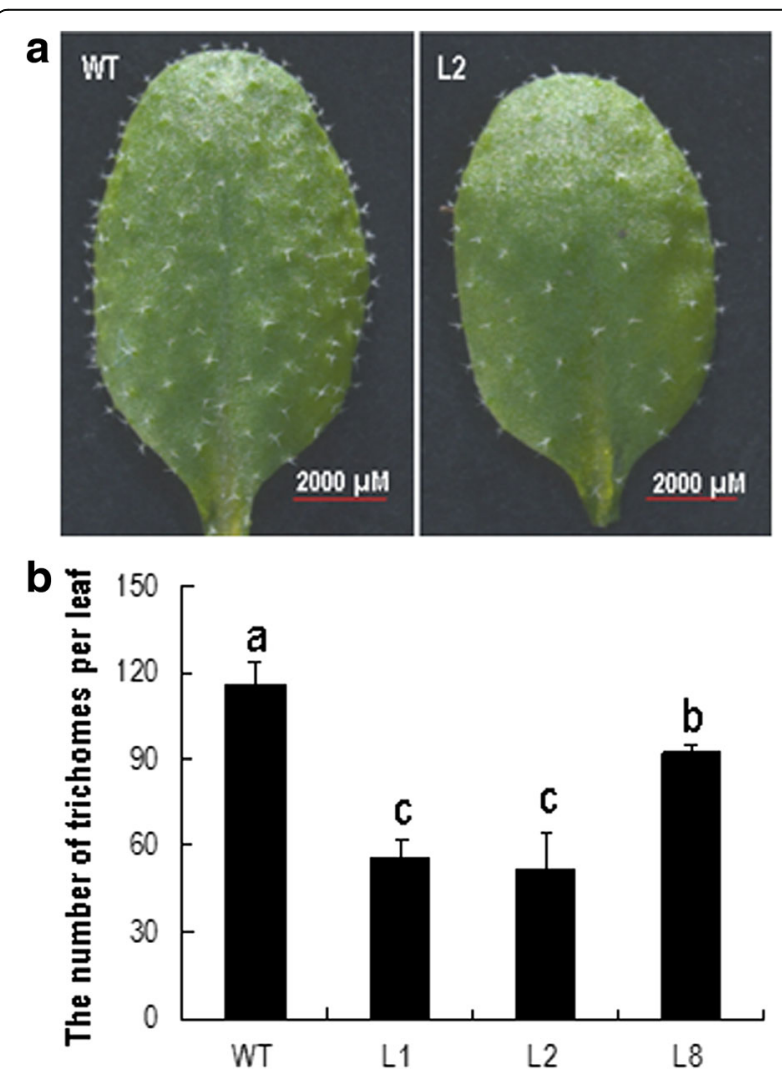

Fig. 7 The morphology and number of leaf trichomes in the wild-type (Col-0) and GhKIS13A1-OX lines. a, Representative leaf images from the wild-type and the $\mathrm{L} 2$ line showing the number and distribution of trichomes. $\mathbf{b}$, The number of leaf trichomes in the wild-type and three GhKIS13A1-OX lines. Different lower-case letters indicate significant differences among different lines at the 5\% probability level (One-way ANOVA, Least Significant Difference test) 
Table 1 Percentages of leaf trichomes with different number of branches in the wild-type and the three GhKIS13A1-OX lines

\begin{tabular}{lllll}
\hline & \multicolumn{4}{l}{ No. of trichome branches } \\
\cline { 2 - 5 } & 5 & 4 & 3 & 2 \\
\hline WT & 0 & $4.2 \mathrm{a}$ & $94.7 \mathrm{a}$ & $0.8 \mathrm{c}$ \\
L1 & 0 & $2.7 \mathrm{c}$ & $85.4 \mathrm{c}$ & $11.3 \mathrm{a}$ \\
L2 & 0 & $2.0 \mathrm{c}$ & $86.1 \mathrm{c}$ & $12.0 \mathrm{a}$ \\
L8 & 0 & $2.7 \mathrm{~b}$ & $93.3 \mathrm{~b}$ & $3.4 \mathrm{~b}$ \\
\hline
\end{tabular}

Data represent the mean percentages of trichomes with the indicated number of branches on the eighth rosette leaf from seven plants. Data were analyzed by one-way ANOVA. Different lower-case letters indicate significant differences among different lines at the $5 \%$ probability level (One-way ANOVA, Least Significant Difference test)

\section{GhKIS13A1 is able to rescue the defective trichome phenotype of the Kinesin-13a-1mutant}

To further confirm the function of GhKIS13A1, we carried out genetic complementation experiment by transforming the homozygous Kinesin-13a-1 mutant with GhKIS13A1 driven by the $35 \mathrm{~S}$ promoter. In the three GhKIS13A1-COM lines (C3, C7 and C10), the number of trichomes was significantly reduced compared with the mutant plants (Fig. 8), and was negatively correlated with their corresponding GhKIS13A1 expression levels, consistent with the results observed in the GhKIS13A1OX lines. Additionally, unlike the Kinesin-13a-1 mutant, which had most of its trichomes with four branches, the GhKIS13A1-COM lines had majority of their trichomes with three branches, and the percentages of such trichomes (93.0-94.9\%) were similar to that of the wildtype $(94.7 \%)$. As a result, the GhKIS13A1-COM lines had an overall branching pattern of trichomes similar to the wild-type (Table 2). These results suggest that GhKIS13A1 is able to rescue the trichome defects of the Kinesin-13a-1 mutant. Overall, the data observed in the transgenic plants indicated that GhKIS13A1 and AtKinesin$13 A$ has a similar function in regulating trichome development in Arabidopsis, but overexpression of GhKIS13A1 in Arabidopsis has a negative effect on the total number of trichomes.

\section{Discussion}

Kinesins are involved in a diverse number of cellular functions. It is expected that Kinesins found in cotton fiber have important cellular roles in cotton fiber development. Four cotton Kinesin genes previously reported to be associated directly or indirectly with cytoskeletons were thought to be related to fiber development [16-19]. We previously cloned 7 cDNAs encoding Kinesins in G. hirsutum [22]. Among them, GhKIS13A1 was chosen for functional analysis. GhKIS13A1 is a central motor domain Kinesin and its full length cDNA is 2730 bp encoding 909aa. Our results showed that GhKIS13A1 has

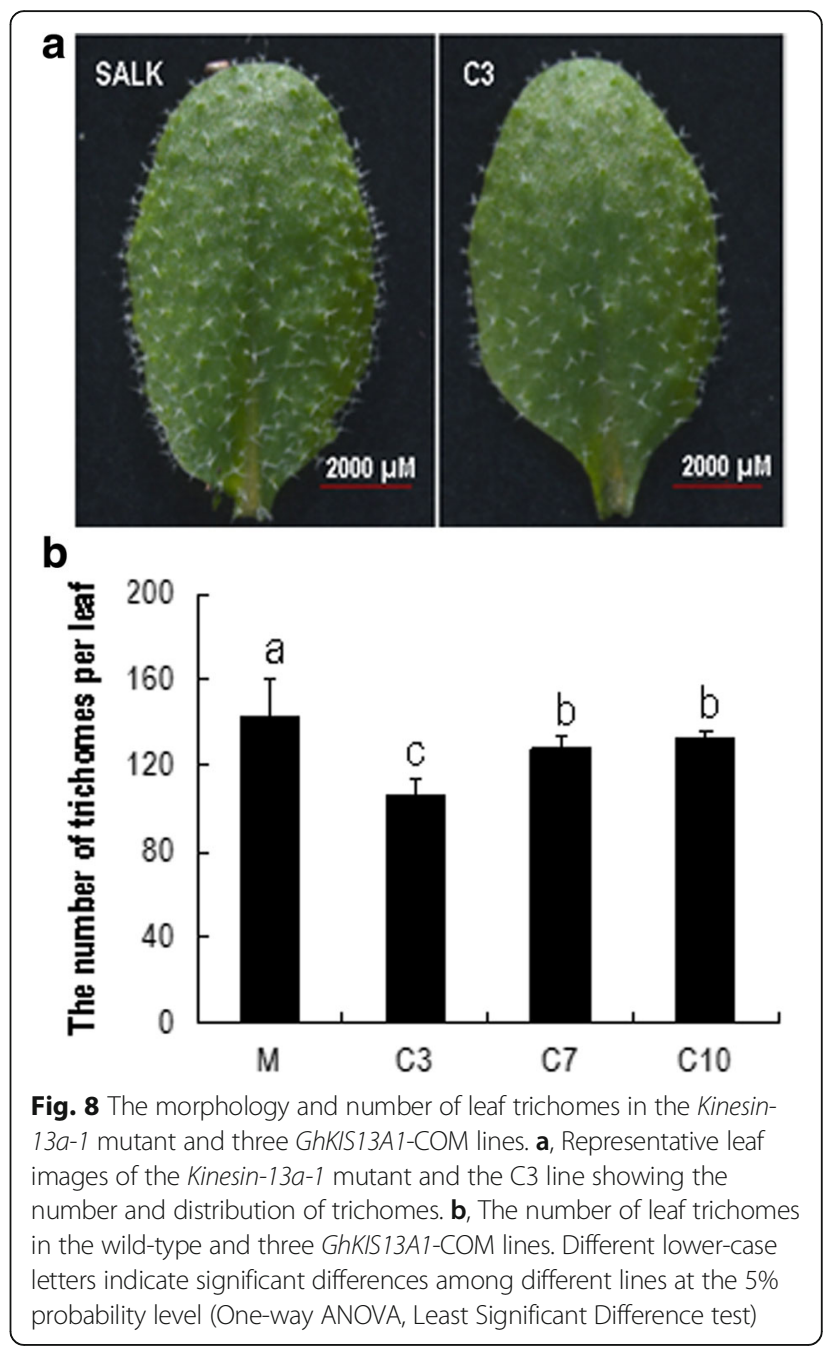

microtubule-activated ATPase activity and microtubules binding activity similar to conventional Kinesins. GhKIS13A1 exhibited a relatively low basal ATPase activity under the condition of without MTs, and its ATPase activity was enhanced by approximately 3 -fold in the

Table 2 Percentages of leaf trichomes with different number of branches in the Arabidopsis Kinesin-13a-1 mutant and the three GhKIS13A1-COM lines

\begin{tabular}{lllll}
\hline & \multicolumn{4}{l}{ No. of trichome branches } \\
\cline { 2 - 5 } & 5 & 4 & 3 & 2 \\
\hline Mutant & $1.4 \mathrm{a}$ & $89.3 \mathrm{a}$ & $8.7 \mathrm{~b}$ & $0.7 \mathrm{a}$ \\
C3 & $0 \mathrm{~b}$ & $3.4 \mathrm{~b}$ & $94.9 \mathrm{a}$ & $1.7 \mathrm{a}$ \\
C7 & $0 \mathrm{~b}$ & $4.0 \mathrm{~b}$ & $94.4 \mathrm{a}$ & $1.7 \mathrm{a}$ \\
C10 & $0 \mathrm{~b}$ & $6.4 \mathrm{~b}$ & $93.0 \mathrm{a}$ & $0.9 \mathrm{a}$ \\
\hline
\end{tabular}

Data represent the mean percentages of trichomes with the indicated number of branches on the eighth rosette leaf from seven plants. Data were analyzed by one-way ANOVA. Different lower-case letters indicate significant differences among different lines at the $5 \%$ probability level (One-way ANOVA, Least Significant Difference test) 
presence of MTs. However, the 3-fold was much lower than other prokaryotic-expressed plant Kinesins and many animal Kinesins [18, 28-30]. The ATPase activity of GhKCH2 was found to be enhanced by 10 -fold in the presence of MTs [18]. The Kinesins must be firstly attached to microtubules, ATP is then hydrolyzed, phosphate is released, and the Kinesins greatly reduced affinity for microtubules [29]. Therefore, much research found that AMP-PNP, the non-hydrolyzable ATP analogue, could enhance the binding of Kinesins to microtubules [30, 31]. GhKIS13A1 cannot be co-precipitated with microtubules in the presence of ATP, and can be coprecipitated with microtubules in the presence of AMPPNP. However, some Kinesins can also co-precipitate with microtubules in the presence of ATP or in a nucleotidefree state $[18,32]$. To our knowledge, this is the first report describing the biochemical characterization of a cotton Kinesin.

A number of genes with a role in the regulation of trichome number and branches have been isolated in Arabidopsis. Loss-of-function mutants of some of these genes showed glabrous leaves or a decease number of leaf trichomes [33-35], but for some genes, their loss-offunction mutants showed an increased clustering of trichomes [36, 37]. In addition, disruption of some genes caused trichomes with abnormal number of branches [38-40]. In this study, we showed that Arabidopsis plants overexpressing GhKIS13A1 exhibited less number of trichomes than the wild-type. We also showed that the Kinesin-13a-1 mutant harboring GhKIS13A1 also had less number of trichomes but had the majority of its trichomes with a restored three-branch morphology. These data suggest that the expression of GhKIS13A1 can inhibit the tichome growth of Arabidopsis, and can be a potential candidate for synchronously regulating the number and branching pattern in plants.

Cotton fibers are single-celled, non-glandular hairs of epidermal. Fiber initiation and fiber elongation are fairly synchronous on each ovule and progresses for 20-30 days [1]. Expression pattern analysis showed that GhKIS13A1 was expressed at a lower level in cotton fiber (containing only trichomes), and maintained a relatively lower level during fiber initiation and elongation stages (Fig. 2). The result suggested that the lower expression of GhKIS13A1 may be beneficial to the fiber initiation and elongation. The hypothesis is in line with the results that the lower expression level of GhKIS13A1 produced more trichomes per leaf in six transgenic lines of Arabidopsis. It is speculated that GhKIS13A1 may negatively regulate the cotton fiber development.

Both Arabidopsis trichomes and cotton fibers are single-celled, non-glandular hairs of epidermal. A number of studies have suggested that there are developmental similarities between cotton fibers and Arabidopsis trichomes [41]. Arabidopsis has been served as a useful model plant for dissecting the mechanisms controlling cotton fiber development. Many Arabidopsis homologous genes have been isolated from cotton, and some of them have been shown to play a similar role in trichome formation in Arabidopsis [42-44]. In this study, the expression pattern analysis and the investigation of the trichome phenotype of Arabidopsis harboring GhKIS13A1 may shed insight into the possible roles of GhKIS13A1 in cotton fiber development. The GhKinesin13A1, whose sequence is identical to the GhKIS13A1, is localized to Golgi stacks, which are frequently associated with microtubules and actin microfilaments [19]. GhKIS13A1 may thus play a similar regulatory role in trichome morphogenesis through its effects on the organization of Golgi stacks. This paper firstly functionally characterized a Kinesin gene from cotton that may be pivotal in fiber development.

\section{Conclusions}

In this study, biochemical characterization showed that GhKIS13A1 has microtubule binding activity, basal ATPase activity and microtubule-activated ATPase activity. Arabidopsis overexpressing GhKIS13A1 exhibited less trichomes than the wild-type, and the Kinesin13a-1 mutant harboring GhKIS13A1 showed less trichomes and had majority of trichomes with restored three-branch morphology, and the percentage of such trichome was matched with that in wild-type. These data suggest that GhKIS13A1 is involved in the trichome development by regulating the total trichome number and branching. It is proposed that GhKIS13A1 may also involve in the regulation of cotton fiber development.

\section{Abbreviations}

AMP-PNP: 5'-adenylylimidodiphosphate; ATP: Adenosine triphosphate; BSA: Bovine serum albumin; DPA: Day post anthesis; GTP: Guanosine

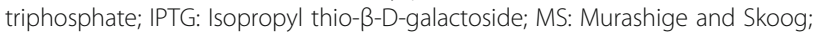
MTs: Microtubules; ORF: Open reading frame; Pi: Inorganic phosphate; qPCR: Real-time quantative PCR; SDS-PAGE: Sodium dodecyl sulfatepolyacrylamide gel electrophoresis

\section{Acknowledgments}

The seeds of the Kinesin-13a-1 mutant of $A$. thaliana were kindly provided by Dr Bo Liu from University of California at Davis. We thank Prof. Wang YZ and Zhu $\mathrm{QH}$ for insightful comments on the manuscript.

\section{Funding \\ This research was supported by the National Natural Science Foundation of China (Grant No: 31301394) and the National Key Research and Development Program (Grant No: 2016YFD0101900). \\ Availability of data and materials \\ The data set supporting the results of this article are available in the NCBI protein (https://www.ncbi.nlm.nih.gov/nuccore) repositories, KP036626.}

\section{Authors' contributions}

$Y J L$ acquired the funding, performed partial experiments and wrote the manuscript. SHZ performed partial experiments and analyzed data. JS supervised the research group. XYZ and LJZ contributed to preparation for the experiment. YCL and FX revised and enhanced the paper. All authors reviewed and approved the final manuscript. 


\section{Competing interests}

The authors declare that they have no competing interests.

\section{Consent for publication}

Not applicable.

Ethics approval and consent to participate

Not applicable.

\section{Publisher's Note}

Springer Nature remains neutral with regard to jurisdictional claims in published maps and institutional affiliations.

\section{Author details}

${ }^{1}$ The Key Laboratory of Oasis Eco-agriculture, Agriculture College, Shihezi University, Bei 4 Road, Shihezi 832003, Xinjiang, China. ${ }^{2}$ College of Life Sciences, Shihezi University, Bei 4 Road, Shihezi 832003, Xinjiang, China.

\section{Received: 17 March 2017 Accepted: 5 June 2017}

\section{Published online: 12 June 2017}

\section{References}

1. Kim HJ, Triplett BA. Cotton fiber growth in plant and in vitro: models for plant cell elongation and cell wall biogenesis. Plant Physiol. 2001;127(4):1361-6.

2. Ruan YL, Llewellyn DJ, Furbank RT. Suppression of sucrose synthase gene expression represses cotton fiber cell initiation, elongation, and seed development. Plant Cell. 2003;15(4):952-64.

3. Li XB, Fan XP, Wang XL, Cai L, Yang WC. The cotton ACTIN1 gene is functionally expressed in fibers and participates in fiber elongation. Plant Cell. 2005:17(3):859-75.

4. Zhang $M$, Zheng XL, Song SQ, Zeng QW, Hou L, Li DM, Zhao J, Wei Y, Li XB, Luo M, Xiao YH, Luo XY, Zhang JF, Xiang CB, Pei Y. Spatiotemporal manipulation of auxin biosynthesis in cotton ovule epidermal cells enhances fiber yield and quality. Nat Biotechnol. 2011;29(5):453-8.

5. Xu B, Gou JY, Li FG, Shangguan XX, Zhao B, Yang CQ, Wang LJ, Yuan S, Liu CJ, Chen LY. A cotton BURP domain protein interacts with a-expansin and their co-expression promotes plant growth and fruit production. Mol Plant. 2013;6(3):945-58

6. Han LB, Li YB, Wang HY, Wu XM, Li CL, Luo M, Wu SJ, Kong ZS, Pei Y, Jiao $G L$, Xia GX. The dual functions of WLIM1a in cell elongation and secondary wall formation in developing cotton fibers. Plant Cell. 2013;25(11):4421-38

7. Schliwa M, Woehlke G. Molecular motors. Nature. 2003;422(6933):759-65.

8. Hirokawa N, Noda Y, Tanaka Y, Niwa S. Kinesin superfamily motor proteins and intracellular transport. Nat Rev Mol Cell Biol. 2009:10(10):682-96.

9. Verhey KJ, Meyer D, Deehan R, Blenis J, Schnapp BJ, Rapoport TA, Margolis B. Cargo of Kinesin identified as JIP scaffolding proteins and associated signaling molecules. J Cell Biol. 2001;152(5):959-70.

10. Sakai T, Honing HV, Nishioka M, Uehara Y, Takahashi M, Fujisawa N, Saiji K, Seki M, Shinozaki K, Jones MA, Smirnoff N, Okada K, Wasteneys GO. Armadillo repeat-containing Kinesins and a NIMA-related kinase are required for epidermal-cell morphogenesis in Arabidopsis. Plant J. 2008;53(1):151-71.

11. Vanstraelen M, Inze D, Geelen D. Mitosis-specific Kinesins in Arabidopsis. Trends Plant Sci. 2006;11(4):167-75.

12. Miki H, Okada Y, Hirokawa N. Analysis of the Kinesin superfamily: insights into structure and function. Trends Cell Biol. 2005;15(9):467-76.

13. Reddy AS, Day IS. Kinesins in the Arabidopis genome: a comparative analysis among eukaryotes. BMC Genomics. 2001;2(1):2

14. Vale RD. The molecular motor toolbox for intracellular transport. Cell. 2003; 112(4):467-80

15. Richardson DN, Simmons MP, Reddy AS. Comprehensive comparative analysis of Kinesins in photosynthetic eukaryotes. BMC Genomics. 2006; $7(1): 18$.

16. Preuss ML, Delmer DP, Liu B. The cotton Kinesin-like calmodulin-binding protein associates with cortical microtubules in cotton fibers. Plant Physiol. 2003;132(1):154-60.

17. Preuss ML, Kovar DR, Lee YR, Staiger CJ, Delmer DP, Liu B. A plant-specific Kinesin binds to actin microfilaments and interacts with cortial microtubules in cotton fibers. Plant Physiol. 2004;136(4):3945-55.

18. Xu T, Qu Z, Yang X, Qin X, Xiong J, Wang Y, Ren D, Liu G. A cotton Kinesin GhKCH2 interacts with both microtubules and microfilaments. Biochem J. 2009;421(2):171-80.
19. Lu L, Lee YR, Pan R, Maloof JN, Liu B. An internal motor Kinesin is associated with the Golgi apparatus and plays a role in trichome morphogenesis in Arabidopsis. Mol Biol Cell. 2005;16(2):811-23.

20. Kitagawa K, Kurinami S, Oki K, Abe Y, Ando T, Kono I, Yano M, Kitano H, Iwasaki Y. A novel Kinesin 13 protein regulating rice seed length. Plant Cell Physiol. 2010;51(8):1315-29.

21. Wei L, Zhang W, Liu Z, Li Y. AtKinesin-13A is located on Golgi-associated vesicle and involved in vesicle formation/budding in Arabidopsis root-cap peripheral cells. BMC Plant Biol. 2009;9(1):138.

22. Zhao L, Xue F, Zhu SH, Li YJ, Liu YC, Sun J. Cloning and expression analysis of GhKinesin13 subfamily genes in Gossypium hirsutum. Acta Agron Sin. 2015;41(4):539-47 (in Chinese with English abstract).

23. Jiang JX, Zhang TZ. Extraction of total RNA in cotton tissues with CTAB-acidic phenolic method. Cotton Sci. 2003;15(3):166-7 (in Chinese with English abstract).

24. Li XY, Wang HQ, Xu T, Cao QH, Ren DT, Liu GQ. Molecular cloning, expression and biochemical property analysis of AtPK1, a Kinesin gene from Arabidopsis thaliana. Chinese Sci Bull. 2007:52(10):1338-46.

25. LeBel D, Poirier GG, Beaudoin AR. A conveninent method for the ATPase Assay. Anal Biochem. 1978;85(1):86-9.

26. Wise AA, Liu Z, Binns AN. Three Methods for the Introduction of Foreign DNA into Agrobacterium. Methods Mol Biol. 2006;343:43-53.

27. Clough SJ, Bent AF. Floral dip: a simplified method for Agrobacteriummediated transformation of Arabidopsis thaliana. Plant J. 1998;16(6):735-43.

28. Umeki N, Mitsui T, Umezu N, Kondo K, Maruta S. Preparation and characterization of a novel rice plant-specific Kinesin. J Biochem. 2006;139(4):645-54.

29. Matthies HJG, Baskin RJ, Hawley RS. Orphan Kinesin NOD lacks motile properties but does possess a microtubule-stimulated ATPase activity. Mol Biol Cell. 2001;12(12):4000-12.

30. Kuznetsov SA, Gelfand VI. Bovine brain Kinesin is a microtubule-activivated ATPase. Proc Natl Acad Sci U S A. 1986;83(22):8530-4.

31. Vale RD, Reese TS, Sheetz MP. Identification of a novel force-generating protein, Kinesin, involved in microtule-based motility. Cell. 1985;42(1):39-50.

32. Alonso MC, Drummond DR, Kain S, Hoeng J, Amos L, Cross RA. An ATP gate controls tubulin binding by the tethered head of Kinesin-1. Science. 2007; 316(5821):120-3.

33. Zimmermann IM, Heim MA, Weisshaar B, Uhrig JF. Comprehensive identification of Arabidopsis thaliana MYB transcription factors interacting with R/B-like BHLH proteins. Plant J. 2004;40(1):22-34.

34. Payne $C T$, Zhang F, Lloyd AM. GL3 encodes a bHLH protein that regulates trichome development in arabidopsis through interaction with GL1 and TTG1. Genetics. 2000;156(3):1349-62.

35. Zhang F, Gonzalez A, Zhao M, Payne CT, Lloyd A. A network of redundant bHLH proteins functions in all TTG1-dependent pathways of Arabidopsis. Development. 2003:130(20):4859-69.

36. Wada T, Tachibana T, Shimura Y, Okada K. Epidemal cell differentiation in Arabidopsis determined by a Myb homolog, CPC. Science. 1997;277(5329): 1113-6.

37. Hulskamp M, Misra S, Jurgens G. Genetic dissection of trichome cell development in Arabidopsis. Cell. 1994;76(3):555-66.

38. Buschmann H, Hauptmann M, Niessing D, Lloyd CW, Schaffner AR. Helical growth of the Arabidopsis mutant tortifolia2 does not depend on cell division pattens but involves handed twisting of isolated cells. Plant Cell. 2009:21(7):2090-106.

39. Nakamura M, Hashimoto TA. Mutation in the Arabidopsis gamma-tubulincontaining complex causes helical growth and abnormal microtubule branching. J Cell Sci. 2009;122(Pt 13):2208-17.

40. Kong Z, Hotta T, Lee YR, Horio T, Liu B. The \{gamma\}-tubulin complex protein GCP4 is required for organizing functional microtubule arrays in Arabidopsis thaliana. Plant Cell. 2010:22(1):191-204.

41. Guan XY, Yu N, Shangguan XX, Wang S, Lu S, Wang LJ, Chen XY. Arabidopsis trichome research sheds light on cotton fiber development mechanisms. Chinese Sci Bull. 2007;52(13):1734-41.

42. Shangguan XX, Yang CQ, Zhang XF, Wang LJ. Functional characterization of a basic Helix-Loop-Helix (bHLH) transcription factor GhDEL65 from cotton (Gossypium hirsutum). Physiol Plant. 2016;158(2):200-12.

43. Guan $X$, Pang $M$, Nah $G$, Shi $X$, Ye W. miR828 and miR858 regulate homoeologous MYB2 gene functions in Arabidopsis trichome and cotton fibre development. Nut Commun. 2014:5(1):3050.

44. Wang S, Wang JW, Yu N, Li CH, Luo B, Gou JY, Wang LJ, Chen XY. Control of plant trichome development by a cotton fiber MYB gene. Plant Cell. 2004;16(9):2323-34. 